\title{
Polar decomposition of Mueller matrices for 2D-structured surfaces
}

\author{
J. M. Sanz , P. Albella, F. González, J. M. Saiz and F. Moreno \\ University of Cantabria, Group of Optics, App. Phys. Dept., Avda. Los Castros s/n, Santander (Spain)
}

\begin{abstract}
In this research, the Polar Decomposition (PD) has been applied to the Mueller matrices (MMs) of the light scattered by linear ribs of rectangular profile on a flat substrate. Although photo-lithographic technique produces a silicon surface, metallic character is acquired by sputtering with gold some of the samples. With a dual rotating compensator polarimeter the MMs are obtained by Fourier Transform analysis. The samples have been numerically modeled by using both FDTD and Extinction Theorem (ET) and MMs have been computed from the results. The scattering depends strongly on the geometry and composition of the ribs, and this sensitivity is noticed for instance in $M_{11}$ element. But information offered by PD parameters is shown to be more apprehensible, like the substrate-induced depolarization or the retardance associated to the rib width.
\end{abstract}

\section{Introduction}

The Mueller matrix (MM) of a system characterizes its physical behaviour for an incident light beam and contains all the scattering information. The angle dependence of this matrix is often included by transforming the scattering matrix elements in plots. Although the bulk of the polarimetry information is completely contained in the MM, both the meaning of its elements and their links often remains obscure. This is why some methods have been proposed, over the past few years, to simplify the physical interpretation of the matrix formalism by introducing a reduced group of appropriate (independent) parameters [1]. One of these methods, the Polar Decomposition method (PD) [2], reduces the number of parameters required to study different optical systems [3]. However, analyzing MM by making use of the PD is not yet customary in light scattering articles.

In a previous research [4], we proposed an application of the PD to simple systems formed by isolated spheres of size smaller than the incident wavelength. We showed that the description of a typical system can be improved by analyzing the behaviour of PD parameters able to reproduce the evolution of real systems and easy to understand from a physical point of view.

Non invasive characterization of small structures, either isolated or on substrate, alone or as a variety of systems, has been focus of interest in the past [5] and is becoming more attractive since structured surfaces are being proposed for its many application in photonics [6], detection [7] or solar cell performance [8], to mention a few.

Since the scattering theory by regular particles, like spheres or cylinders, is broadly developed [9] and applied many of systems and configurations (see for instance [10]) it is necessary to develop a procedure to compute and ana-

a e-mail: sanzjm@unican.es lyze in comprehensible terms other geometries of increasing complexity.

In this work we use numerical simulations to analyze the theoretical behaviour of linear elevations of rectangular profile on a flat substrate (ribs). The MMs of the samples were measured by using a Dual Rotating Compensator Polarimeter (PDRC) [11] developed in our labs. Non-zero values of theoretically null MM elements are observed and analyzed by means of PD. This data processing shows the dependence of the depolarization parameters on the substrate and, what it is more important, allows us to summarize the polarimetric information of a system in the lowest number of parameters, being aware of the physics of the problem.

\section{Experimental set-up}

\subsection{PDRC polarimeter}

The PDRC polarimeter [11] developed in our labs consists of a He:Ne laser, a Polarization State Generator (PSG), a sample holder, a Polarization State Analyzer (PSA) and a Detector. PSG is made up of a polarizator and a rotating quarterwave plate and, the PSA is made up of a rotating quarterwave plate and an analyzer. A long-focal length lens is placed after the laser, in order to control the spot size and beam divergence. The sample is on a rotating stage in order to control the angle of incidence, and the PSA-Detector couple is mounted on the arm of a rotor to select the scattering angle. Sample holder is on a tilt rotating platform with a XYZ Nanopositioner (fig. 1 shows a schematic vision of the PDRC). A Fourier Transform analysis allows to extract the $\mathrm{MM}$ of the sample for each scattering angle [11].

This is an Open Access article distributed under the terms of the Creative Commons Attribution-Noncommercial License 3.0, which permits unrestricted use, distribution, and reproduction in any noncommercial medium, provided the original work is properly cited. 
EPJ Web of Conferences

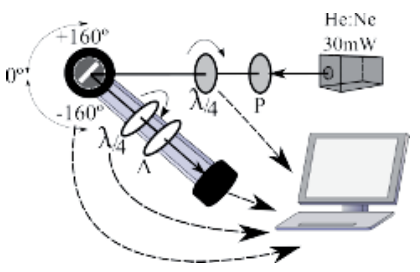

Fig. 1. Experimental set-up.

\subsection{Samples}

The samples consist of linear ribs (rectangular profile) on a flat substrate. These 2D-geometries have been used previously [12], and respond to the need of obtaining a configuration with strong interference, diffraction and/or multiple scattering effects. Two arrays of samples were made by photo-lithographic techniques on a silicon substrate. The metallic character is given by gold sputtering. The ribs width vary from 1 to $4 \mu \mathrm{m}$ (height: $1 \mu \mathrm{m}$ ), and the internal separation between ribs range from 1 to $5 \mu \mathrm{m}$. Fig. 2 shows a SEM image and the scattering geometry scheme.

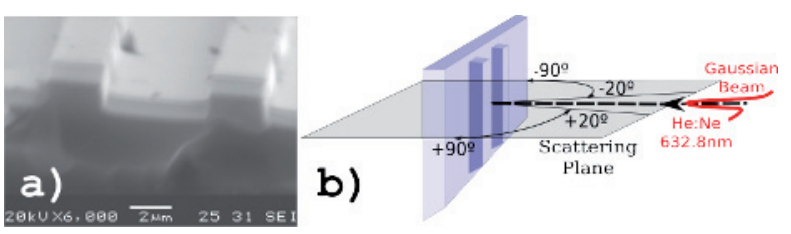

Fig. 2. Two-rib: (a) SEM image x6000 and (b) scattering scheme.

\section{Numerical model}

Extinction Theorem (ET) [13] is a computational method applied to solve scattering problems (particularly those with 2D-structures). On the other hand, Finite Differences Time Domain (FDTD) [14] is a powerful tool to solve 2D and 3D-structures without restrictions (apart from those involving the required computational resources). Our ET results for a 2D-geometry, consisting of a section of a linear Sirib on a flat substrate, were tested with the corresponding FDTD results obtained for a 3D model of the same problem. As fig. 3 shows, ET and FDTD produce similar patterns.

\subsection{Geometry analysis}

The inverse scattering problem of spherical or cylindrical geometries located on substrates (for which the main parameter is the section radius, $R$ ) is today very well characterized [15]. For the rectangular rib two section parameters, height and width, must be specified. In order to know the behaviour of these geometries, it is necessary to analyze how the theoretical scattering pattern, in normal incidence, changes with the rib dimensions. By using ET, we

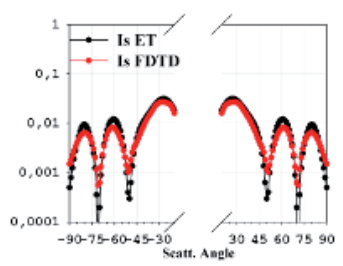

Fig. 3. Comparison between 2D-ET and 3D-FDTD: Is scattering pattern for a Si-rib $(0.9 \times 0.9 \mu \mathrm{m}, \lambda=633 \mathrm{~nm})$

numerically obtain the parameter $M_{11}$ for a set of systems, in which the height or width of a single Au-rib $(1 \times 1 \mu \mathrm{m})$ is slightly changed. Results were plotted in fig. 4, and show strong changes in the angular distribution of $M_{11}$ for small height variations, with shifts or even absence of some minima, while width variations produce smaller changes.
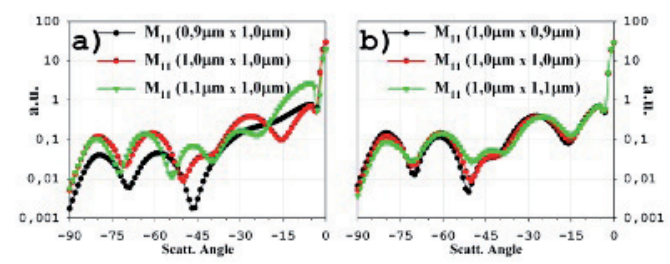

Fig. 4. $M_{11}$ Scattering pattern for an Au-rib: (a) height variations, and (b) width variations.

These changes in the scattering pattern are indicators of a strong dependence on the size of the rib, even for a perfect rectangular shape. Such perfection is not expected form the experimental samples, after fabrication and sputtering processes. Furthermore, in order to reproduce, to some extent, results, theoretical ribs have to be redimensioned, therefore constituting a fitting procedure for the object itself, and an inverse problem procedure. Small deviations in the maximum position may come partly from the sample profile (fig. 5(b)), which presents a slope greater than $0.05 \mu \mathrm{m} / \mathrm{mm}$ (for a laser spot of approx. $1 \mathrm{~mm}$ in the sample). In addition, smoothed internal corners and sample fabrication defects (fig. 2) contribute to local shape of the rib.
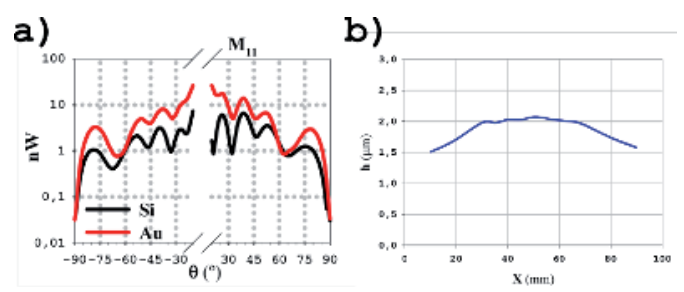

Fig. 5. (a) One rib $(1 \times 3 \mu) M_{11}$ pattern. (b) Height variation along the array of samples. 


\subsection{Polar decomposition algorithm}

The scattering matrices obtained in experimental measures have been post processed with an algorithm that performs the PD. After testing the physical reliability of the matrices [16], it was found that, in all the cases analyzed, the MMs obtained were reliable and the application of PD algorithm is justified. Once measured, the MM can be decomposed by using a forward PD as an equivalent product of a diattenuator matrix $\left(M_{D}\right)$, an elliptical retarder matrix $\left(M_{R}\right)$ and a depolarizator matrix $\left(M_{\Delta}\right)[17]$ :

$$
M=M_{\Delta}\left(d_{i}, a_{i}, z_{i}\right) M_{R}(\varphi, \delta, \rho) M_{D}\left(\alpha, \beta, t_{1}, t_{2}\right), i=1,2,3
$$

where $\alpha, \beta, \varphi$ and $\rho$ are the azimuths and ellipticities of, respectively, the diattenuator and retarder; $t_{i}$ is the transmittance along the $i$ axis of the diattenuator, $\delta$ is the phase of the retarder and the depolarization matrix can be described by:

$$
M_{\Delta}=\left(\begin{array}{cc}
1 & \mathbf{0}^{T} \\
\mathbf{P}_{\Delta} & m_{\Delta}
\end{array}\right), \text { where } m_{\Delta}=\left(\begin{array}{lll}
d_{1} & a_{1} & a_{2} \\
a_{1} & d_{2} & a_{3} \\
a_{2} & a_{3} & d_{3}
\end{array}\right), \mathbf{P}_{\Delta}=\left(\begin{array}{c}
z_{1} \\
z_{2} \\
z_{3}
\end{array}\right)
$$

As we will later comment PD analysis show that, in our measures, only six parameters show a visible contribution to the MM: the total transmittance for unpolarized light (parameter $M_{11}$ without normalization), the principal depolarization coefficients $d_{i}$, the phase parameter $\delta$ and the transmittance along one of the diattenuator axes, that is related with the other axis (normalized to the total transmittance) by $t_{1}=1-t_{2}$. Another magnitude of interest is the depolarization $(\Delta)$ that can be obtained from the principal depolarization coefficients by using ec. 3 :

$$
\Delta=1-\frac{\left|d_{1}+d_{2}+d_{3}\right|}{3}
$$

\section{Results}

\subsection{General MM analysis}

Angular evolution of MM parameters is shown in fig. 6 for single square ribs $(1 \times 1 \mu \mathrm{m})$ of $\mathrm{Si}$ and the same with Au sputtering. Although angular symmetry is determined by normal incidence, some asymmetry features indicate the presence of some geometrical asymmetry in the sample (either from fabrication or from the sputtering itself). In second place, elements within the up-right and downleft $2 \times 2$ boxes should be null for a 2D-geometry when working in a plane of incidence normal to de rib axis [9], but the values of those elements at some angles are too far from zero. Previous works [12] put the blame on polarimeter imperfections and alignment, but in our case PDRC has been tested previously showing an error margin lower than $1 \%$. As for the diagonal boxes, it seems obvious that the sputtering process has a smoothing effect on the patterns.

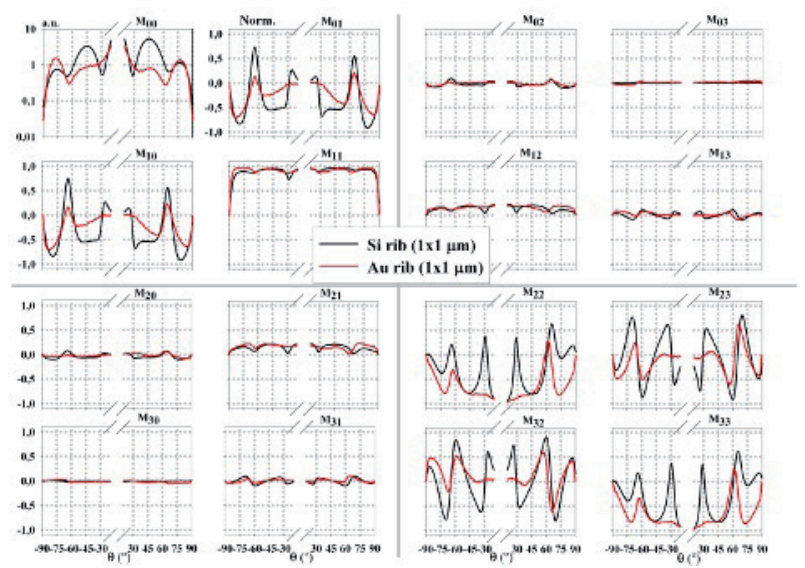

Fig. 6. Mueller matrix evolution with scattering angle for a rib $(1 \times 1 \mu \mathrm{m})$ of Si (black line) and $\mathrm{Au}$ (red line).

\subsection{PD analysis: influence of the substrate}

Once MM goes through $\mathrm{PD}$, the resulting $M_{\Delta}$ matrix contains three non-zero elements, $d_{i}(i=1,2,3)$. These elements show a similar behaviour (though $d_{1} \gtrsim d_{2} \gtrsim d_{3}$ ). When the depolarizing components, summarized in depolarization $\Delta$, are weighted by the total transmittance (or total scattering), it represents the amount of depolarized light. This product is shown in fig. 7(a) for a single $1 \times 1 \mu$ rib of either Si or Au-sputtered. Apart from the difference introduced by the sputtering process in the angular distribution of depolarization, it is noticeable that an important asymmetry appears around the specular (normal) direction. The following question arises from these results: Is the substrate responsible for any of those features? Lets remind that the cross-section of a rib is much smaller than the area of the spot in the sample, this being a classical situation in scattering by structured surfaces. To answer this, both substrates were measured, MM obtained and PD performed, producing the scattering shown in fig. 7(b) and the amount of depolarization plotted in fig. 7(c). It is interesting to observe that, though the sputtered surface is more efficient in producing scattered light, the stronger depolarizing component of the etched Si compensate for the difference producing similar amounts of depolarized light. The angular plot of the ratio, red dots in fig. 7(d), shows that sputtering is more efficient in producing depolarization than the etched Si surface underneath, except for observation near normal, where the smoother Si becomes more efficient. As for the asymmetry, it seems to concentrate around the specular direction, as was the case for the rib. This asymmetry stands for any small variation around normal incidence (a few arc-min. must be kept for proper operation), thus showing that surface roughness contains some skewness component produced during the etching process that also affect the measurements of the ribs. This was confirmed after repeating the measurements with sample turned $180^{\circ}$. 

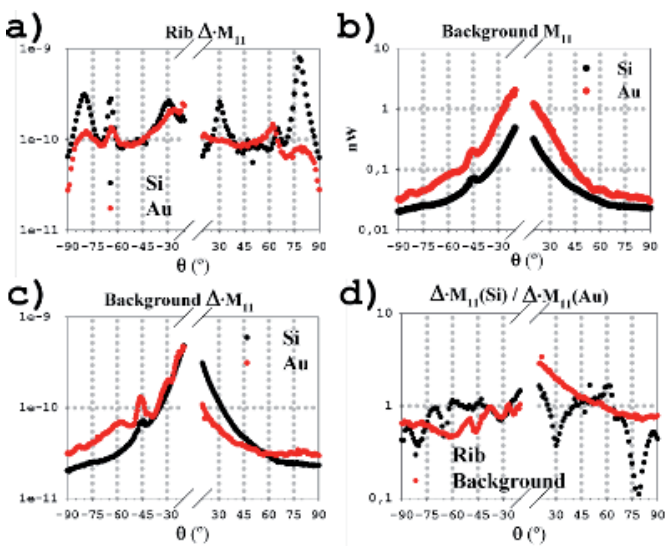

Fig. 7. (a) 1 rib $(1 \times 1 \mu)$ depolarization weighted, (b) background transmittance, (c) background depolarization weighted, and (d) ratio of depolarization weighted $(\mathrm{Si} / \mathrm{Au})$.

\subsection{Behaviour of other PD parameters}

Once depolarization effects have been extracted from the $\mathrm{MM}$, information about pure polarization component can be analyzed. As an example, fig. 8 shows PD parameters pattern for two Si-ribs $(1 \times 3 \mu \mathrm{m}, 1 \mu \mathrm{m}$ apart $)$. Azimuthal and ellipticity parameters on retarder $(\varphi$ and $\rho)$ are null, while retardance ( $\delta$, not unwrapped in the plots) exhibits an interesting pattern (see fig. 8(b)), that correlates with the diattenuation $t_{1}$ (fig. 8(a)). a)

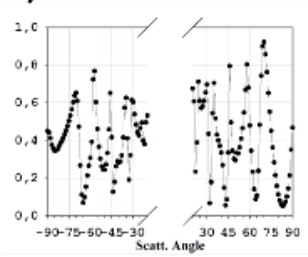

b)

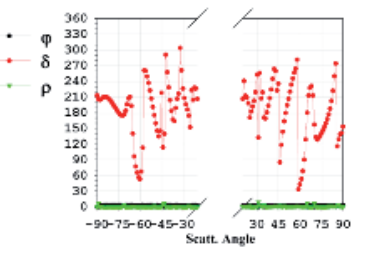

Fig. 8. Two Si-rib $(1 \times 3 \mu \mathrm{m}, d=1 \mu \mathrm{m})$ : (a) $t_{1}$ and (b) retarder PD parameters vs. scattering angle ( $\delta$-phase is not unwrapped).

Retardance patterns are closely related to the lobes often shown in the literature for the intensity patterns (e.g. fig. 5(a)) associated to this kind of samples, and often refereed to as phase functions, used, among other things, for sizing purposes. As it is shown in fig. 9, the number of abrupt dephase changes increases with the rib width for both $\mathrm{Si}$ and $\mathrm{Au}$. However, oscillations in Au-samples are smoother than Si-ones, and this effect could be due to two causes: different Au-film height on sputtering deposition due to surface affinity, which may reduce the effective size of the rib, and/or Au intrinsic effects on polarization.

\section{Conclusions}

Numerical results shows the possibility of detecting small changes in the size and shape of objects by directly observ-

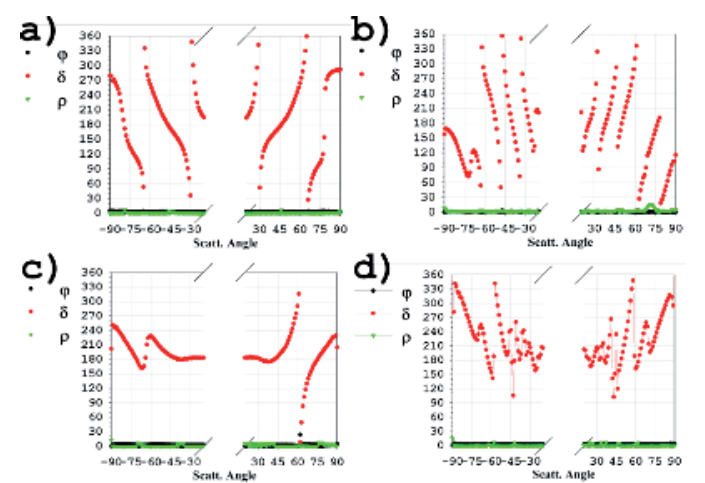

Fig. 9. Retarder $\mathrm{PD}$ parameters vs. scat. angle for a rib: (a) $\mathrm{Si}$ $1 \times 1 \mu \mathrm{m}$, (b) Si $1 \times 3 \mu \mathrm{m}$, (c) Au $1 \times 1 \mu \mathrm{m}$, and (d) Au $1 \times 3 \mu \mathrm{m}$.

ing parameter $M_{11}$ of the MM. This sensitivity, however, does not bring much insight into the physics of the scattering problem. Experimental measurements show that processes suffered by the sample affect very strongly the polarimetric observation. Results in PD formalism are better summarized in terms of parameters with a physical meaning. Furthermore, PD can separate substrate induced depolarization from sample pure polarimetric information, thus pointing out the kind of experimental advantages of PD formalism when the complexity of the depolarizing system increases.

\section{Acknowledgements}

This research has been supported by the Ministry of Education of Spain under project FIS2007-60158 and by USAITCA through R\&D 1390-PH-01.

\section{References}

1. R. Ossikovski, JOSA A 25(2), 473 (2008)

2. S.Y.L. et al., JOSA A 13(5), 1106 (1996)

3. J.J. Gil, Eur. Phys. Jour. Appl. Phys. 40, 1 (2007)

4. J.M.S. et al., JQSRT 110, 1369 (2009)

5. F.G. et al., Light Scattering from Microstructures (Springer, 2000)

6. E. Ozbay, Science 311, 189 (2006)

7. T.K. et al., Phys. Rev. Lett. 95 (2005)

8. K.R.C. et al., Opt. Exp. 16(26), 21793 (2008)

9. H. Hulst, Light scattering by small particles (1981)

10. P.A. et al., JQSRT 109(8), 1339 (2008)

11. R.M.A. Azzam, Opt. Lett. 2(6), 148 (1978)

12. V.J.I. et al., App. Opt. 26, 1799 (1987)

13. F.G. et al., JQSRT 70(4-6), 383 (2001)

14. K.S. Yee, IEEE Trans. Ant. Prop. 14(3), 302 (1966)

15. J.L.P. et al., Part. Part. Syst. Charact. 16(3), 113 (1999)

16. S.R. Cloude, Optik 75(1), 26 (1986)

17. R.O. et al., Opt. Lett. 32(6), 689 (2007) 